\title{
Five Shapes of Cognitive Dissonance - Using Objective Hermeneutics to Understand the Meat Paradox
}

\author{
Stefan Mann ${ }^{1}$ iD $\cdot$ Helene Renaux ${ }^{2}$
}

Accepted: 12 October 2021 / Published online: 15 November 2021

(c) The Author(s), under exclusive licence to Springer Nature Switzerland AG 2021

\begin{abstract}
Objective Hermeneutics is a qualitative method that focuses on few sequences of texts, which helps understand single cases. It is used to explore how consumers cope with the contradiction between their enjoyment for meat and their empathy for animals without using frameworks drafted by social scientists. Five cases are analysed, which range from strong references towards the societal norm of meat eating to a feeling of uncertainty in the face of the animals' death. None of the cases, however, sees the necessity to find a rationale for their own meat consumption. The study concludes by raising the question about the persistence of a societal consensus for meat consumption.
\end{abstract}

Keywords Qualitative methods · Text analysis · Consumer attitudes · Switzerland

\section{Introduction}

The meat paradox - the contradiction between our empathy for animals and our preference for meat (Hestermann et al., 2020) - is a case for a likely cognitive dissonance that is not an under-researched subject. In recent years, the scientific encounter with this phenomenon has strongly been intensified. Four strains of literature have emerged. One includes papers which, by trying to find solutions for either individuals (Ursin, 2014), the media (Lewis, 2018) or governments (Bastian and Loughnan, 2016) in handling meat consumption, develop normative arguments. Related to that, a second group of papers does not take a normative stance itself but develops likely positions of how individuals may cope with their cognitive dissonance (Aaltola, 2019; Hestermann et al., 2020; Rothgerber, 2020). On the empirical side, a third group in the literature applies such frameworks in surveys, identifying to which degree they apply among different samples of consumers (Piazza et al., 2015; Buttlar and Walther, 2018; Dowsett et al., 2018; Camilleri et al., 2020). Moreover, the fourth group largely uses qualitative methods to distinguish types of justifications for

On behalf of all authors, the corresponding author states that there is no conflict of interest.

Stefan Mann

stefan.mann@agroscope.admin.ch

Agroscope, Tänikon 1, CH-8356 Ettenhausen, Switzerland

2 ETH Zurich, Rämistr. 101, CH-8093 Zurich, Switzerland 
meat eating in different cultural contexts (Oleschuk et al., 2019; Panagiotou and Kadianaki, 2019; Khara et al., 2021).

While this study has the closest link to the fourth group of qualitative, empirical research, it uses the method of Objective Hermeneutics to follow a more inductive and, regarding text analysis, a more microscopic approach than the latter. Reasons for this methodological choice and a brief introduction to this method will be provided in Sect. 2. Section 3 is then devoted to the presentation and interpretation of five cases of meat eaters, before Sect. 4 discusses the findings in the context of popular theoretical concepts and Sect. 5 concludes the study.

\section{Method}

\section{The Choice of Objective Hermeneutics}

In the four strains of literature that have approached the meat paradox as described above, scientists have found different theoretic options of how to handle the meat paradox and, while allowing for cultural differences, they attempt to check to which degree these options apply.

While this description of past research is certainly oversimplistic, it is still true that a hypothesis-driven deductive approach has dominated, and it may be worthwhile to confront this with an analysis that is distinctly inductive. Qualitative research in general has been described to be suitable for a rigorous inductive (Gioia et al., 2012) and explorative (Hollstein, 2011) way of gathering information.

However, the heterogeneity of qualitative methods is considerable, and not all methods are equally suitable for the purpose of inductive and explorative research. Coding, for example, which is used in methods like Grounded Theory (Thornberg and Charmaz, 2014) or Content Analysis (Neuendorf and Kumar, 2016), is an example for a methodology in which pre-defined theories may play a prominent role. Marathe and Toyama (2018), for example, show how codes may emerge from existing conceptual theories.

Objective Hermeneutics is among the most suitable choices for research that is unanimously inductive (Borcsa, 2016). It also is a method that has high ambitions with respect to the analysis of single cases and low ambitions in terms of generalizability. In fact, a lot of the literature applying Objective Hermeneutics deals with the analysis of a single case (Lee and Kim, 2014; Naumann, 2019). A thorough and unbiased understanding of these cases is more important for scholars using Objective Hermeneutics than any generalizability.

For each case, Objective Hermeneutics works with a case-specific hypothesis describing the social substance that is supposed to come increasingly closer to social reality while more information is available (Wernet, 2019). The starting point is some background information about the case, sometimes even involving the use of genograms (Hildenbrand, 2004). This may allow first guesses about expected patterns, which then are to be confronted with the empirical material.

The empirical material is usually analysed in a group so that individual perceptions about the meaning of single words or phrases can be put into the context of other perceptions. The process of consensus building during the interpretation by the different group members (or the "rule-based method of group interpretation", as Pflugmacher (2016; 285) calls it) is supposed to bring the "objectivity" into hermeneutics. While the authors, for example, are meat eaters with a desire to become vegetarian and partly even published 
about a 'post-lethal agriculture' (Mann, 2020), other members of the group, vegetarians or meat eaters without regret, may have neutralized one-sided interpretations by the authors in interpreting the sequences.

The specific way in the analysis of the text also contributes to the desired objectivity. Any "summaries" of the things said are avoided because they would too strongly include the subjectivities of the ones who summarize. Instead, only two or three sequences of maybe a quarter or half a page that contribute to the research question are chosen and regarded as "protocols of reality" (Wernet, 2000). These protocols are then used for a joint microscopic analysis in which it is attempted to extract the objective meaning of what is said. This is accomplished by putting single phrases into different contexts in which such phrases are likely to be heard. Numerous examples for this will be provided in Sect. 3 . Subsequently, the joint characteristics of these different contexts or narratives in which a sequence is likely to be heard provide the bracket that describes the "objective" meaning of the phrase.

Only such a thorough analysis of (a small part of) the empirical material is able to uncover the latent, and often subconscious, structure of sense within the case at stake. This opens up the possibility to dive more deeply into the anatomy of the meat paradox.

\section{Data Collection and Analysis}

In the international literature on meat consumption, it is well known that men eat about twice as much meat as women do (Prättälä et al., 2007; Arganini et al., 2012; Uzmay and Cinar, 2017) and different reasons for this imbalance have been explored (DeLessio-Parson 2017; Dieterle 2015). As it thus can be assumed that the meat paradox among men may be more prevalent than among women, it was decided to limit the cases to meat-eating men, and as the study was carried out in the German-speaking part of Switzerland, sampling was in fact confined to meat-eating men living in Switzerland and speaking German. To allow for a sufficiently thorough analysis of the single cases, five men were interviewed. The selection of cases borrowed the method of contrasting sampling from methods like Grounded Theory (Dey, 2004), so that a large bandwidth of scope of the meat paradox could be accounted for. The contrasts included age, origin and educational level.

The interview aimed to approach rationales of individual dietary (meat) choices but it also was important to avoid unnecessary pressure which could have resulted into statements of social desirability (Kaushal, 2014; Przyborski and Wohlrab-Sahr, 2014). Therefore, interviewees were asked to give an interview about meat consumption, not about vegetarian or vegan diets. The interviews started by the interviewer generally requesting the respondents to state what comes to their minds when thinking about meat. If then only very general statements were coined (such as media coverage of meat consumption), people were asked about their personal approach to meat consumption. If respondents expressed concerns about their own meat consumption, they were asked why they would not be vegetarian (which certainly was a situation which might have caused some unwanted moral framing of the interview). Within this frame, the interviews flowed relatively freely, deepening the points the respondents raised. They lasted between 45 and $90 \mathrm{~min}$. Their arrangement was made more difficult because they took place in Winter 2020/21 when Switzerland, as most other countries, imposed strict meeting and hygiene conditions due to COVID-19.

While four of the five interviews were held in Swiss German, they were transcribed in written German. Selected sequences, as is the best practice for Objective Hermeneutics, 
were then analysed in group work, so that a common understanding about their 'objective' content could emerge.

\section{Sequence Analysis}

The five subsequent cases, as they had to put in any order, were placed according to the degree of problems they stated with their own meat consumption. Space only allows a single sequence of each respondent, although some more were analysed during the project.

\section{Blurring Normativity}

$\mathrm{T}$ is a 59-year-old single, part-time tram driver and is also a small entrepreneur for highend equipment. It had been somewhat difficult to convince him for the interview, but he finally agreed because he was told about a peer also agreeing to be interviewed. The interview was scoped by T's tendency to digress from the subject, so the interviewer's main concern was to frequently take the interview back to the issue of meat consumption. The chosen sequence comes from the end of the interview after the respondent had stated that he could neither be attracted to become a vegetarian not to try meat substitutes.

I: Because you like too much to eat it? Or why?

T: That is how it should be. I don't know. I just like to have a piece of meat. I appreciate it. This is enjoyment for me. It is not simply 'yes I have to every day'. There is folk that needs to every day. That actually is not what I have to. It could also be that I skip it for a whole week.

\section{I: $M h m$}

T: So there is no problem in foregoing it once. But I simply have it and then I take it.

As every question, the interviewer's question at the beginning of the sequence opened a space for expected answers. She suggested a possible reason for not becoming a vegetarian. This suggestion may be perceived by $\mathrm{T}$ as provocative for two reasons. One is that she suggested that not ethical considerations, but rather mere sympathy for eating meat, could be the reason for a decision that could also be answered on moral grounds. The other is the use of the adverb "too", which contains a normative connotation in itself. The interviewer may want to say that sympathy for the taste of meat is an evil in itself. This strong request to "defend" meat consumption is reinforced by the subsequent "or why". A why always demands a justification, so the strong request of a moral defence of T's meat consumption becomes obvious.

It seems that this request for normativity is fulfilled by $\mathrm{T}$ when he replies that "es gehört sich", a German term that is difficult to translate. "Es gehört sich" refers to the catalogue of good manners. Typical connections of the term would be to use a fork for eating or to pay respect to elders; thus, the translation "that is how it should be" appears to be the best fit. Apparently, the respondent shared our analysis that the interviewer's question demanded a strong moral statement. After the claim that eating meat was part of good behaviour, however, one should expect an argument why this should be the case. This argument is not delivered. Instead, there is a statement of missing knowledge. In colloquial language, such statements are common when one wants to emphasize the possibility of error and some openness for other positions. In our context, however, the statement is mainly used 
to transgress as quickly from normativity as the subject had been entered. This implies that the claim of a norm in favour of meat consumption remains empty.

What follows now are three statements declaring preferences for meat. The first of them may be the most interesting as it contains three additional features. One is the filler word "just" which makes the statement more "matter of fact" like. The second is the emphasis on having instead of eating, a reference to the property rather than to the act of consumption. Finally, T does not plainly refer to meat as such, but to a piece of meat. A piece is even further away from the living body than meat itself, a good example for the tendency towards abstraction that is often used when talking about harsh concrete realities like in the language of war (Gay, 1999). Therefore, all three additions contribute to deflect attention from the act of eating parts of another body. The second and third statement describing T's enjoyment of meat consumption do not need this structuring framework any more, and they are more plainly hedonic statements, eventually emphasizing subjectivity ("for me").

$\mathrm{T}$ then confronts this hedonism with the patterns of addiction. He refers to an attitude that, by omitting the central verb, resembles the language of sexuality ("I have to every day"). After he caricatures such addictive positions (opened with an admitted "Yes"), he becomes more elaborate, referring to "folk" that suffer from such addictions, again omitting the subject of eating meat. The choice of these omissions, which we also know from the realm of sexuality, may uncover two parallels between practicing sexuality and eating meat. The one to which T refers is addiction. The other, however, may also be guilt. Uttering "eat meat" may as much be avoided as uttering "have sex", as both may somehow fall in a sphere of guilty pleasures and societal taboos.

Towards the end of the phrase, the respondent undermines his non-addiction. Again, he uses three sentences to make this point. In the first, he sets himself aside from the group of the addicted. This claim is illustrated by the second sequence, in which he draws the image of the possibility of not consuming "it" (the term "meat" is still avoided) for an entire week. The third of these sequences is used to emphasize the ease of skipping meat for one meal, which sounds much less hypothetical than the possibility of a meatless week.

The last sentence, in a sense, summarizes the avoidance of any serious normative position towards eating meat, even though the term "meat" is still not used. The availability of meat is used as the key factor for "taking" it. Although, literally, it would be impossible to take something that you already have, T's point becomes obvious. Beyond the two facts that meat is available and that its taste appeals to T, no further reasoning is needed.

\section{Emphasizing Hedonism}

$\mathrm{H}$ is a 30 -year-old Hungarian-born $\mathrm{PhD}$ student at a major Swiss university. During the interview taking place at H's office, $\mathrm{H}$ is curious to find out about the interviewer's position about eating meat, which is, however, only revealed at the end. The following sequence is taken from the middle of the interview:

I: Now you have said already so much. How is it then for yourself? What does meat mean to you? (...) Or how do you consume meat, to remain concrete?

$\mathrm{H}$ : So I like food in general. I would now myself, so I also like meat. I am (...) I can understand the arguments of 'Yes it is not really environmentally friendly'. Er, I would also eat meat menus in the cafeteria or elsewhere, but it is not that I have to eat meat necessarily. So if there is a good alternative, I can also eat and choose it, and sometimes it is good.

(...) - longer pause 
The interviewer's input, in this sequence, has much more room than in the first. The first remark may seem misplaced in an interview where it is the whole point that the respondent says so much. It may contain the hidden message that the quantity of transmitted information does not match the added value for the interviewer. The subsequent two questions then clarify that the interviewer aims for a personal statement from $\mathrm{H}$, while they differ in terms of depth. While "how is it" rather refers to the emotional level, the reference to the meaning of meat rather refers to a philosophical level.

All this does not result in an immediate answer. Although it became apparent that $\mathrm{H}$ likes to talk, this seems to come to an end as soon as the subject becomes personal. No response is provided. After some pause, the interviewer adds another question. The "how do you consume" can be answered in a more technical way than the questions asked before. It liberates $\mathrm{H}$ from the necessity to reflect about the inner self and its relation to meat consumption.

Nevertheless, the respondent chooses to make a statement about his personal preferences in the answer. Notably, however, the opening statement is not related to meat. The "so" (a German 'also') points to the necessity to develop a broader context to make oneself understandable. This context consists of the fact that H likes "food in general". Why could this be of importance in the context of meat consumption? Most likely because it prioritizes enjoyment over any other consideration within food choices and tries to weaken the importance of meat.

After this introductory remark, the sequence becomes less straightforward. While we hear that meat is not an exception to the rule that $\mathrm{H}$ enjoys food, two incomplete sentences catch the reader's attention. "I would now myself" apparently struggles for some self-identification. Such an identification would have to go towards a non-vegetarian, as $\mathrm{H}$ was asked about meat consumption and $\mathrm{H}$ likes meat as a product. However, $\mathrm{H}$ decides to avoid such a categorization. After the statement that $\mathrm{H}$ likes meat, the same happens again. "I am" is the typical beginning of some self-classification. However, there seems to be some reason why $\mathrm{H}$ sheds back from putting himself into the box of omnivores.

Some possible reason is provided by the following sentence. After a purely emotional part about food that provides enjoyment, a purely cognitive one follows. H would not, however, argue himself that eating meat harms the environment. He is only willing to follow the rationale of the ones who do so, or rather, to the ones talking to third parties and agreeing to them with an affirmative "yes". In fact, he does not agree with them straightaway that meat consumption harms the environment but rather agrees that it is not really (!) environmentally friendly. The need to acknowledge the detrimental environmental effects of meat consumption is affirmed in the most indirect way possible.

$\mathrm{H}$ returns from this unpleasant mission by finally answering I's question about his personal consumption patterns. However, $\mathrm{H}$ would not state that he eats meat in the cafeteria. He would eat it, and not primarily the meat that was the core of I's question, but the meat menu. In this part of the sequence, some obvious parallels to T pop up. While $\mathrm{T}$ would eat a piece of meat, instead of meat itself, $\mathrm{H}$ prefers the meat menu. Both need to generate some cognitive distance between meat as such and their preference.

The obvious parallels to $\mathrm{T}$ continue when, subsequently, $\mathrm{H}$ emphasizes that he is not addicted to eating meat. In the last sentence of the sequence, there is a noteworthy normative element supposed to support H's non-addiction. H can (although not necessarily does) choose (and then even eat) the alternative to the meat menu, but only provided it is good. Even in this case, the good alternative apparently often turns out to not be as good as promised, and only in some cases, the good alternative is finally good. 


\section{Unlimited Freedom}

S, a 24-year-old student of financial management who is active in the country's Liberal Party prefers to hold the interview with a mask (in winter 20/21). He was the only respondent totally unknown to the interviewer before the interview took place; so the atmosphere was somewhat more distant. The following sequence is taken from the end of the interview.

I: And that is why I had previously asked the question why, so how due to which decisions or which factors do you decide to choose meat if you have different options or mmh what causes that you buy meat from this butcher?

S: Mhm.

I: Yes.

$\mathrm{S}$ : Yes this really is a good question and I have thought about it several times, but I do not come to a very smart answer. Probably like three things. First, I believe it is generally difficult for people to separate from habits, I believe. That definitely applies to me. If I became used to something, I have difficulties to detach from it. I believe this is certainly something influencing my behaviour in this realm. Maybe not so strongly. Second, I believe that in spite of everything it is still enjoyment for me to be able to eat a piece of meat. Er, so with that I refer to from preparation to er (...) for example if I have invited guests. I simply find that a piece of meat is, it sounds awkward, but is (...) appealing food, if it is prepared accordingly. And the third point is strongest for me, er, the third point that would influence or which would be a reason why I would not want to skip meat consumption in the future, which is: personally I do not like to restrict myself.

The interviewer's question certainly has something coercive. It becomes clear that the same question had be asked before (apparently without being answered). The interviewer does neither leave it with a single question, but rather asks two, nor with the simple interrogative "how" which she makes more implicit by referring to the underlying decisions. The reference to the availability of different choices clarifies that it is about S's decisions, whereas that "this butcher" makes it clear that a rich narrative had been exchanged.

Despite the coercive nature of the questions posed, $\mathrm{S}$ answers with an affirmative Mhm. While such a statement could be expected after hearing a story or a statement, it is rather unexpected as an answer for a question and reveals considerable reluctance to answer maybe also because he needs time to reflect. The interviewer reacts by a "yes". This affirms the questions asked, indicates that she is not going to ask any different question instead and plays the ball back to $S$.

In the answer that is finally coined, $\mathrm{S}$ ignores the fact that the interviewer had asked at least two questions, acknowledging that the underlying question-why would S not skip meat-had become obvious. He does not only refer to 'this question' but also classifies it as good, which usually refers to a high relevance of the topic, but then takes a step that leads to a particular epistemic position. As for the other respondents looked at, it is usually common sense that we are able to reflect about our own decisions and can put them into a rational and consistent framework. This is implicitly denied by $\mathrm{S}$ when he indicates that he would be unable to find a "very smart answer" to motivate his meat consumption. This strategy of distancing one's cognition from personal behaviour is maintained in the rest of the phrase, by often using words like "I believe" or "maybe". In terms of self-analysis, S remains essentially ignorant and implicitly postulates that our decisions go beyond our own rationality. Yes, S may consume meat, but no, there is no certainty about the reason for it. 
Nevertheless, after this disclaimer, a system of three different points is elaborated that should motivate S's meat consumption. His first 'belief' concerns the power of habits, stated in a de-personalized form first, before $\mathrm{S}$ admits that this applies to himself, although 'maybe not so strong'. While this reference to societal norms may resemble T's reference to 'how it should be', his second point-hedonism-is even closer to T's and in particular to H's rationale. While his reference to 'a piece of meat' rather than meat is a noteworthy parallel to the previous two cases, the main difference on the explanation of meat hedonism is S's reference to guests. His enjoyment does not only range to his own gustatory pleasure, but extends to the process of preparing it for others.

Why would S say something that, according to his own statement, sounds awkward? If there was an additional need to illustrate the nature of cognitive dissonance, it is here, between the pure sympathy for the taste of meat - provided the preparation was fine-and the knowledge that there would be more to food choices than hedonism.

The third and allegedly most important point for meat consumption by $\mathrm{S}$, however, is an unwillingness to restrict himself, i.e. liberalism on an individual level and, therefore, a new aspect. The introduction of this point is made in a rather long-winded sentence. It is important to him that one does not lose sight of the system of list composition and its objective. While he reminds the interviewer again that the three points would influence his behaviour, he then corrects himself and labels the points as reasons. This tension refers back to the epistemic problem of understanding oneself. Despite all uncertainties claimed by $\mathrm{S}$, he has eventually found reasons for his own behaviour.

By citing liberalism as the most important point for $\mathrm{S}$ to maintain meat consumption, he lifts the issue to a different level. It draws the attention away from the question if animals should be killed to serve as food toward the question if any practice in society should be restricted. Liberalism is an approach that aims to maintain as many liberties of the individual in a society. It would be an entirely appropriate argument if the interviewer would have dealt with the question whether to ban meat consumption in a society. Taken together with the epistemic claim that one does not understand one's own choices, S deals with himself like a liberal state with its citizens: it does not judge his own choices, but lets them act freely. In this respect, S implicitly follows a school of thinking that attaches several selves to individuals, for example, the distinction between market preferences and reflective preferences by Brennan and Lomasky (1983) or between first and second order preferences by Frankfurt (1992).

\section{Obeying Rules}

$\mathrm{R}$ is a 60-year-old retired engineer who is strongly engaged in social projects in his neighbourhoods. As the interview took place in his neighbourhood, the interview was often disrupted by acquaintances coming by. The following sequence is taken from the beginning of the interview and is a reaction of $\mathrm{R}$ on the opening question to tell something about meat consumption.

R. On the one hand, I try to only buy meat which is reduced. Meat produced in Switzerland that is reduced, because it is overaged in the shop, so because it expires in the shop.

That's what I buy. I thus have restricted myself, that the meat will not be destroyed, right? But (...) yes, like yes, I simply, I like meat, but I stop liking it if it er, (...) yes if it breaks all like rules, yes. Don't you have more questions? (Laughs)

I: Yes, yes. 


\section{$\mathrm{R}$. It is difficult.}

$\mathrm{R}$ is the only respondent for whom the question about meat consumption in general suffices, and he begins talking about his own consumption patterns after a while. Introducing a rule with "on the one hand" indicates that $\mathrm{R}$ intends to introduce a set of two rules. It is telling, however, that this first rule, to only buy reduced meat, is a rule that $\mathrm{R}$ tries to obey. It is likely that this wording is chosen because sometimes $\mathrm{R}$ may fail to act according to the rules he sets for himself. While the concept of the split self resembles that of $\mathrm{S}$, the difference is that $\mathrm{S}$ intends to be liberal with himself, whereas $\mathrm{R}$ is trying to set limits.

Although the "other hand" is not introduced explicitly, it can be assumed that the second rule that meat should be domestically produced. This is not a rule, however, which $\mathrm{R}$ tries to motivate. Instead, the first rule takes much more mental room in what has to be considered as R's justification for eating meat. It is not sufficient for R to paraphrase his attempts to save meat from waste three times; it is also necessary for him to emphasize that "that's what I buy". While the informational content of this message is small, it underlines that R's behaviour is shaped by a clear conviction.

The unfinished next sentence, starting with a 'but', could go into two different directions. It could either emphasize that no additional animal will be killed for $\mathrm{R}$, as he only saves meat from being wasted, or it could draw attention to the bright side of meat consumption. The latter option may be supported by the fact that this is what happens after the pause. Thus, it needs a few attempted starts before $\mathrm{R}$ is ready to admit that he likes meat. These attempts include a "simply" which often is used to avoid too deep reflections.

At this stage, the 'but' which went unfinished beforehand comes to a completion. It is obvious that meat is not an acting subject that is able to keep and break rules. However, $\mathrm{R}$ uses the image of meat as a subject that is usually good but sometimes hurts the rules. This avoids real-life complexities of the different actors including his own responsibility as a consumer in the meat chain, where it can well be that an animal was raised well and that retailers follow best practices, but that the slaughterhouse had complications. It is therefore not surprising that R's rules are not just rules, but more vague "like rules " ("so Regeln" in the German original). Moreover it enables $\mathrm{R}$ to see meat as a definable subject, which enables him to draw a clear line between himself as a subject and the meat as a subject, similar to the "piece of meat" in T's answer.

At this stage, the most likely continuation of the sequence would be an outline about the rules, maybe in general terms or in a hierarchical order. However, this is not what happens. R changes the level of his conversation and stops talking about meat. The most likely explanation for this sudden shift of levels is that he is not really clear about the very content of his rules in excess of what he has already said. His need for further questions may reveal that $\mathrm{R}$ has reached a dead end in rolling out his rules that, at the end of the day, may be less clear than they should be.

This, at least, is what can be derived from the end of the sequence. "It is difficult" usually follows a discourse about the contradictions or dilemmas that cannot be resolved. This, however, was not mentioned in R's sequence, which rather presented a firm normative framework. The perception of meat consumption as a difficult subject reveals that $\mathrm{R}$ sees some cognitive dissonance, which, however, cannot be easily described.

\section{Conscious About Death}

$\mathrm{P}$ is a 21-year-old skater fascinated by Punk culture. While he has an education in retailing, he carries out different occasional jobs. He is a friend of the interviewer's friend. He 
participated in the interview with a mask. The sequence was taken from the end of the interview after the question why $\mathrm{P}$ would not be vegetarian or vegan. Again, because of the lengthy answer to this question, the sequence does not begin with the question itself.

P: Sometimes I think like, yes look, every time when I eat meat, a critter has simply died.

I: Yes

P: And I eat it now.

I: Yes

P: I cannot do anything about its death, but I find this is already something, simply this consciousness

I: Yes.

P: that is such a critter, it has lived, it maybe has been a mega nice (...) sheep or so (laughs)

I: Yes, right, yes.

P: I don't know, right?

I: Yes

P: If you think about that, then, then (...) no idea, I believe that automatically you'll change your eating habits a bit, but you know, no idea.

The "sometimes I think" at the beginning of the sequence usually points to a subjective thought or a hypothesis. In this case, however, P uses this term to claim a factual connection. Strictly speaking, this factual condition is wrong. Eating meat does not lead to the immediate death of an animal. Literally, however, the connection between killing animals and eating meat is, of course, obvious, even if it is labelled as an occasional thought-a thought to which the eyes ("yes, look") of either the interviewer or P himself still have to be opened, breaking out of the terrain of abstracted language that is often use when talking about meat. Two more details in this sequence should be mentioned: one is the labelling of the animal as critter (or "Vieh" in German). This slightly derogatory term may be chosen to make its death less painful, whereas the "simply" may remind the reader to R's use of this term. In the case of $\mathrm{P}$, the fact that the 'simply' makes more matter-of-factly is the death of an individual animal.

In a way, $\mathrm{P}$ corrects the factual mistake in his sequencing by his second reference to his own eating. If he eats it now, as he says, it starts becoming clear that the killing of the respective animal has occurred earlier. What appears as an unnecessary doubling serves to clarify the logical link between dying and eating.

After this link has been clarified, $\mathrm{P}$ extends it. $\mathrm{P}$ uses his factual separation from the process of slaughtering to, in a way, claim his innocence related to the animal's death. This claim is certainly correct in a factual way, albeit what could follow at this point would be the question whether consumers, by stopping to eat meat, could save the lives of animals. Instead of such or similar elaborations, however, $\mathrm{P}$ terminates the narrative on the impact of his diet and moves onto a meta level by classifying the thoughts he just has made. If anything "is already something", it often describes the first step on a lengthy journey. In this case, P's notion that consciousness may be the first step towards behavioural change is consistent with some social psychological frameworks like 4P (Chance et al., 2014) or AIDA (Lee et al., 2018).

From this excursion to the meta level (from meat consumption towards states of consciousness about meat consumption) and after a brief affirmation by the interviewer, $\mathrm{P}$ moves back to the situation of his meat consumption. In his perception, although the meat is being consumed, the individual animal still exists, even if its individual abilities are first 
depreciated by labelling it plainly as "such a critter". Its ontology has been constituted by the fact that is has lived, and this constitution of an individual apparently outlasts death. While P's sentence begins with an explicit de-individualisation, he now allows for the option of the meat having been an adorable sheep. With this, he leads the narrative about the embeddedness of his meat consumption to an end. While this narrative could easily be perceived as a tragedy, its end is accompanied by laughing. This is in accordance with the fact that humour is a frequent way to cope with contradictions that cannot be resolved (Wilson, 2008).

The sequence ends agnostically. It becomes obvious for $\mathrm{P}$ that he is unable to resolve the apparent contradiction between the "mega nice sheep" and his meat consumption. This issue leads to P's acknowledgement that he does not know, and even this statement urgently ("right?") needs affirmation from the interviewer.

P's cluelessness becomes more explicit in the last two lines of the sequence. P intends to outline which consequences a thorough reflection about the dilemma that he has drawn would have. This sentence, however, ends in silence and, eventually, in a remaining statement of ignorance about the ontological contradiction between the mega nice sheep and the beast. It seems likely that a first impulse would bring $\mathrm{P}$ to switch to a vegetarian diet, but that he is not ready to take this step. This finally leads to a tuned down version of what $\mathrm{P}$ planned to say in the first place. Changing eating habits 'a bit' seems to be a fair compromise between ignoring the deathly consequences of one's consumption patterns and the lust for meat.

\section{Discussion}

A strength of Objective Hermeneutics is that single cases can be understood in depth, and this strength has not fully been played out by the analysis above. Each of the five cases could have been entered in more detail, both by digging deeper in the sequences displayed, and by adding a second or maybe third sequence. However, despite the relatively brief references to the five cases, both commonalities and differences could be detected that would have been difficult to identify with any other analytical tool.

It has been shown by the literature cited in Sect. 1 that there are theoretical pathways to solve the cognitive dissonance created by the meat paradox, such as depicting animals as evil or by denying that animals can feel pain. Authors like Rothgerber (2020) have also shown that people choose such and similar pathways if you suggest it to them in a survey. However, if merely discussing people's meat consumption in a less suggestive framework as done for this paper, none of the five cases chose such pathways, at least not by adapting their view on animals. Apparently, there was no need to solve the meat paradox in front of the interviewer and to draw a consistent image of one's personal behaviour in terms of meat consumption.

Is a cognitive dissonance a cognitive dissonance if no commitment exists to solve it? The fathers of the concept have answered this question in different ways. While Festinger's (1957) original definition of cognitive dissonance did not pay attention to the commitment, the specification of cognitive dissonance theory by Brehm and Cohen (1962) would consider commitment as an essential factor. This definitional issue, however, is not of major importance to understand the nature of the meat paradox. For our context, it is sufficient to know that contemporary psychologists consider 'the way in which the self is involved in dissonance' as 'an important matter' (Mills, 2019; p.53). 
It is possible, however, to find more common ground between the five cases than the absence of attempts to justify animal husbandry. All five respondents enjoy eating meat. Furthermore, all know the societal norms firmly behind them and their eating behaviour. Not everybody is stating it as clearly as the first respondent $\mathrm{T}$ who emphasizes, "this (eating meat) is how it should be". However, the fact that eating meat is clearly part of our culture takes a large part of the burden away to justify something for which not much ethical substance exists. In addition, the special status and the implicit associations of meat as the food of the feast (Zaraska 2016), as mentioned by S, probably makes the acceptance and enjoyment of vegetarian dishes more difficult, as described by H. Hence meat consumption seems to indulge a complex sensory network, which originates from a rich cultural heritage. Some efforts are undertaken, however, to show that their own behaviour is better than that of some others. While T, for example, bitches about the ones that are addicted to meat, P claims that being conscious about one's behaviour is already 'something'.

All meat eaters are well aware that their practice is accepted by society, underlining carnism as the prevailing ideology (Joy und Sezgin 2017). However, different shapes or shades between the cases can well be detected. This applies, for example, to the respondent's rhetoric on death. By transforming meat to either 'a piece of meat' or 'a meat menu', the first three respondents attempt to rather hide the fact that there is a necessary link between killing and meat consumption. The fifth respondent, in contrast, approaches this issue of death much more actively, eventually conceding his ignorance on how to cope with it. The same applies to the detrimental environmental effects of meat consumption that are highlighted by $\mathrm{H}$ without offering any solution or feeling at all involved in the environmental impact of his meat consumption.

There certainly are major differences between the cases in terms of self-management. $\mathrm{R}$ actively attempts, even if not always successfully, to restrict himself to the leftovers of his retailer's meat shelves, giving him the impression that there is no instant relation between his consumption and animal deaths. S, on the other hand, is so liberal that he does not wish to restrict his own behaviour. This is a particularly sophisticated way to eliminate the need to justify anything. Moreover, this worldview enables the distraction of the personal involvement and responsibility for the participant's individual food choices, which have, of course, a daily impact. Another dimension of differences is the level of abstraction that respondents use when talking about meat. While $\mathrm{T}$, for example, carefully increases the abstraction by turning meat into a piece of meat, $\mathrm{P}$ breaks out of the abstraction by explicitly referring to the critter that he eats.

\section{Conclusions and Outlook}

The strength of qualitative research is that connections researchers would not have thought about beforehand open themselves up for analysis (Mann, 2001; Haigh and Miller, 2018). Furthermore, the strength of Objective Hermeneutics is that it makes the most out of only little amounts of data, so that a deep understanding about single cases can be obtained. These strengths have led to new findings on five cases on meat-eating men in Switzerland.

Scientists love rationality because it is their most important tool. In the case of the obvious contradiction between empathy for animals and the sympathy for meat, they look for ways out of the dilemma by creating theoretical concepts that would justify meat consumption. This, however, is not the pathway chosen by the five respondents depicted above. They differ in terms of the dissonance they feel, and while four of them consider meat 
consumption as a problem, they neither had an urgent desire to solve this in the past nor did they perceive the necessity during the interview. Together with the fifth respondent, who most actively claims society on his side, they all feel that eating meat is such an accepted pattern in our world that no need for justification exists.

However, societal consensuses may change over time. While in most parts of the world, homosexuality has changed from an unaccepted issue to an accepted one, slavery has gone the opposite way. While global meat consumption is still on the rise, the share of vegans and vegetarians in the Western world is rising as well, intergenerational friction is starting to show, and the abstracting language with which we are protecting ourselves from the realities of eating dead creatures may collapse. We should be prepared for the case that a broader societal debate about the legitimacy of meat consumption will emerge.

\section{References}

Aaltola, E. 2019. The meat paradox, omnivore's akrasia and animal ethics. Animals 9 (12): 1125.

Arganini, C., A. Saba, R. Comitato, F. Virgili, and A. Turrini. 2012. Gender Differences in Food choice and dietary intake in Modern Western Societies. In Public Health, Social and Behavioral Health, ed. J. Maddock. New York: Books on Demand.

Bastian, B., and S. Loughnan. 2016. Resolving the meat paradox, a motivational account of morally troublesome behaviour and its maintenance. Personality and Social Psychological Review 21 (3): 278-299.

Borcsa, M. 2016. Latent Meaning Structures in Couple Relations, Introducing Objective Hermeneutics into Systemic Therapy Research. In Research Perspectives in Couple Therapy, ed. M. Borcsa and P. Rober. Heidelberg: Springer.

Brehm, J.W., and A.R. Cohen. 1962. Explorations in cognitive dissonance. New York: John Wiley.

Brennan, G., and L. Lomasky. 1983. Institutional Aspects of "Merit Goods" Analysis. Finanzarchiv 41 (2): 183-201.

Buttlar, B.E., and Walther. 2018. Measuring the meat paradox, how ambivalence towards meat influences moral disengagement. Appetite 128: 152-158.

Camilleri, L., P.R. Gill, and A. Jago. 2020. The role of moral disengagement and animal empathy in the meat paradox. Personality and Individual Differences 164: 110103.

Chance, Z., M. Gorlin, and R. Dhar. 2014. Why choosing healthy food is hard, and how to help, presenting the 4P's framework for behaviour change. Customer Needs and Solutions 1: 253-264.

DeLessio-Parson, A. 2017. Doing vegetarianism to destabilize the meat-masculinity nexus in La Plata, Argentina. Gender, Place \& Culture 24 (12): 1729-1748. https://doi.org/10.1080/0966369X.2017. 1395822.

Dey, I. 2004. Grounded Theory. In C. Seale, Qualitative Research Practice. Thousand Oaks, SAGE.

Dieterle, J. M. 2015. Just food. Philosophy, justice, and food. London, Rowman \& Littlefield International.

Dowsett, E., C. Semmler, H. Bray, R.A. Ankeny, and A. Chur-Hansen. 2018. Neutralizing the meat paradox, cognitive dissonance, gender and eating animals. Appetite 123: 280-288.

Festinger, L. 1957. A theory of cognitive dissonance. Stanford: Stanford University Press.

Frankfurt, H. 1992. The faintest passion. Proceedings and Addresses of the American Philosophical Association 66 (3): 5-16.

Gay, W.C. 1999. The Language of War and Peace. In Encyclopedia of Violence, Peace, and Conflict, ed. L.R. Kurtz and J. Turpin. New York: Academic Press.

Gioia, D.A., K.G. Corley, and A.L. Hamilton. 2012. Seeking Qualitative Rigor in Inductive Research, Notes on the Gioia Methodology. Organizational Research Methods 16 (1): 15-31.

Haigh, C., and E. Miller. 2018. What Really Matters to Patients? Digital Storytelling as Qualitative Research. In Cultivating Compassion, ed. P. Hardy and T. Sumner. Heidelberg: Springer.

Hestermann, N., Y. Le Yaonanq, and N. Treich. 2020. An economic model of the meat paradox. European Economic Review 129: 103569.

Hildenbrand, B. 2004. Fallrekonstruktive Familienforschung und Familientherapie, Die Sequenzanalyse in der Genogrammarbeit. Familiendynamik 29 (3): 257-287.

Hollstein, B. 2011. Qualitative Approaches. In The SAGE Handbook on Social Network Analysis, ed. J. Scott and P.J. Carrington. Thousand Oaks: SAGE. 
Joy, M., and H. Sezgin. 2017. Warum wir Hunde lieben, Schweine essen und Kühe anziehen. Karnismus eine Einführung. Münster: Compassion media.

Kaushal, K. 2014. Social desirability bias in face to face interviews. Journal of Postgraduate Medicine 60 (4): 415-416.

Khara, T., C. Riedy, and M.B. Ruby. 2021. A cross cultural meat paradox, a qualitative study of Australia and India. Appetite 164: 105227.

Lee, H.-S., and S.-K. Kim. 2014. A qualitative study on the bicultural experience of second-generation Korean immigrants in Germany. Pacific Science Review 16 (2): 97-109.

Lee, S., H. Song, C. Lee, and J.F. Petrick. 2018. An integrated model of pop culture fan's travel decision making processes. Journal of Travel Research 57 (5): 687-701.

Lewis, K.C. 2018. A Meat Paradox, Media's Role in Mitigating the Omnivore's Dilemma. The University of Southern Mississippi, Master Thesis

Mann, S. 2001. How Do You Find Out What Really Matters for Public Acceptance-The Case of Swine Production Sites in Rural Communities. Forum Qualitative Social Research 2(1) https://doi.org/10. 17169/fqs-2.1.979.

Mann, S. 2020. Could We Stop Killing?-Exploring a Post-Lethal Vegan or Vegetarian Agriculture. World $1(2): 124-134$.

Marathe, M., K. Toyama 2018. Semi-Automated Coding for Qualitative Research, A User-Centered Inquiry and Initial Prototypes. In CHI '18, Proceedings of the 2018 CHI Conference on Human Factors in Computing Systems. https://doi.org/10.1145/3173574.3173922.

Mills, J. 2019. Improving the 1957 version of dissonance theory. In Cognitive dissonance, re-examining a pivotal theory in psychology, ed. E. Harmon-Jones. Washington: APA.

Naumann, M. 2019. Utilization of Resilience in German Development Policy-An Objective-Hermeneutical Analysis Exemplified by the Case of Welthungerhilfe. In Resilience in Social, Cultural and Political Spheres, ed. B. Rampp, M. Endress, and M. Naumann. Heidelberg: Springer.

Neuendorf, K.A., and A. Kumar. 2016. Content Analysis. In The international encyclopedia of political communication. London: Wiley.

Oleschuk, M., J. Johnston, and S. Baumann. 2019. Maintaining meat, cultural repertoires and the meat paradox in a diverse sociocultural context. Sociological Forum 34 (2): 337-360.

Panagiotou, E., and I. Kadianaki. 2019. From cognitive dissonance to cognitive polyphasia, a sociocultural approach to understanding meat paradox. The Theory of Social Behaviour 49 (2): 235-253.

Piazza, J., M.B. Ruby, S. Loughnan, M. Luong, J. Kulik, H.M. Watkins, and M. Seigerman. 2015. Rationalizing meat consumption. The 4Ns. Appetite 91: 114-128.

Pflugmacher, T. 2016. Objektive Hermeneutik. In Empirische Forschung in der Deutschdidaktik, ed. J.M. Boelmann. Hohengehren: Schneider Verlag.

Prättälä, R., L. Paalanen, D. Grinberga, V. Helasoja, A. Kasmel, and J. Petkeviciene. 2007. Gender differences in the consumption of meat, fruit and vegetables are similar in Finland and the Baltic countries. European Journal of Public Health 17 (5): 520-525.

Przyborski, A., and M. Wohlrab-Sahr. 2014. Forschungsdesigns für die qualitative Sozialforschung. In Handbuch Methoden der empirischen Sozialforschung, ed. N. Baur and J. Blasius. Heidelberg: Springer.

Rothgerber, H. 2020. Meat-related cognitive dissonance, A conceptual framework for understanding how meat eaters reduce negative arousal from eating animals. Appetite 146: 104511.

Thornberg, R., and K. Charmaz. 2014. Grounded Theory and Theoretical Coding. In The SAGE Handbook on Social Network Analysis, ed. U. Flick. Thousand Oaks: SAGE.

Ursin, L. 2014. The ethics of the meat paradox. Environmental Ethics 38 (2): 131-144.

Uzmay, A., and G. Cinar. 2017. The likelihood of sheep meat consumption in Turkey. Italian Journal of Food Science 29: 209-221.

Wernet, A. 2000. Einführung in die Interpretationstechnik der Objektiven Hermeneutik. Heidelberg: Springer.

Wernet, A. 2019. Wie kommt man zu einer Fallstrukturhypothese? In Vom Fall zur Theorie, ed. D. Funke and T. Loer. Heidelberg: Springer.

Wilson, N.A. 2008. Was that supposed to be funny? A rhethorical analysis of problems, politics, contradictions in contemporary stand-up comedy. Moines: The University of Iowa Press.

Zaraska, M. 2016. Meathooked. The history and science of our 2.5-million-year obsession with meat. New York: Basic Books.

Publisher's Note Springer Nature remains neutral with regard to jurisdictional claims in published maps and institutional affiliations. 